\title{
Interaction, Protection and Epidemics
}

\author{
Sanjeev Goyal * $\quad$ Adrien Vigier $^{\dagger}$
}

October 14, 2014

\begin{abstract}
Individuals respond to the risk of contagious infections by restricting interaction and by investing in protection. We develop a model that examines the trade-off between these two actions and the consequences for infection rates.

There exists a unique equilibrium: individuals who invest in protection choose to interact more relative to those who do not invest in protection. Changes in the contagiousness of the disease have non-monotonic effects: as a result interaction initially falls and then rises, while infection rates too may initial increase and then decline.

We then consider a society with two communities that differ in their returns from interaction - High and Low. Individuals in isolated communities exhibit different behavior: the High community has a higher rate of protection and interaction, and a lower rate of infection. Integration amplifies these differences.
\end{abstract}

*Faculty of Economics \& Christ's College, University of Cambridge. Email: sg472@cam.ac.uk

${ }^{\dagger}$ Oekonomisk Institutt, University of Oslo. Email: adrien.vigier@uio.econ.no We are grateful to the editor, Erzo Luttmer, and two anonymous referees for comments that have significantly improved the paper. We thank Diego Cerdiero, Julien Gagnan, and Anja Prummer for detailed comments on an earlier draft. We thank Eddie Dekel, Matthew Elliot, Andrea Galeotti, Mark Gersovitz, Chris Gilligan, Jesper Rudiger, Brian Rogers, Pauline Rutsaert, Larry Samuelson, Flavio Toxvaerd, and seminar participants at Cambridge, UCL (Louvain), Oslo and Oxford for useful discussions. We thank the Cambridge-INET Institute for financial support. 


\section{Introduction}

Interactions between individuals generate value, but facilitate the spread of infections. This tension is salient in diseases such as influenza, HIV and tuberculosis, but also applies to the Internet and other digital networks. ${ }^{1}$ In all these examples, infection spreads primarily through interpersonal contacts: so prevalence can be reduced by restricting interaction and/or by investing in protection. This paper develops a model that examines the trade-off between these two courses of action and its consequences for the spread of infections.

In our model, a population faces the risk of becoming infected. Every individual chooses how much to interact with others in the population, and whether or not to protect himself. Interactions generate benefits but increase the risk of infection from infected others. Protection is available, at a fixed cost. The protection rate, the profile of interaction, and the contagiousness of the infection together determine the extent of the disease in the population.

We first establish that a (Nash) equilibrium exists and is unique. For a broad class of circumstances, equilibrium protection rates are interior: only a part of the population protects. Individuals who invest in protection interact more than those who do not. Restricted interaction and protection are substitutes. This relation is consistent with empirical observation. For example, in their well known study on British sexual attitudes and behavior, Wellings et al. (1994) report a positive correlation between the frequency of new partners and the use of condoms.

The contagiousness determines the probability of becoming infected from interacting with

\footnotetext{
${ }^{1}$ There are 3 to 5 million cases of acute influenza and between 250,000 and 500,000 deaths are attributed to this infection, annually. In 2012, over 8.5 million people were infected with tuberculosis and 1.3 million deaths were attributed to it. In the same year, 2.3 million new cases of AIDS were reported and over 1.5 million people died due to the disease; over 36 million people have died due to HIV/AIDS so far (WHO (2013, 2014a, 2014b)).

The Internet reflects a similar tension: on-line interactions generate rewards but may serve as a conduit for the spread of viruses and worms which compromise user value. As energy, communication, travel, consumer interaction increasingly adopt digital networks, cybersecurity has emerged as a major concern. We discuss the relevance of our analysis for cybersecurity later in the introduction.
} 
infected individuals, and is a key parameter in the study of epidemics. ${ }^{2}$ We find that equilibrium response to contagiousness is non-monotonic. There exists a threshold level of contagiousness: below this value, protection rates are zero, and the response to higher contagiousness is through reduced interaction only. This threshold reflects the fixed costs associated with protection: below the threshold incurring the costs is not worthwhile. Above the threshold, returns from protection outweigh costs. Greater contagiousness now induces greater protection, and a first-order stochastic dominance shift in the profile of interaction. Infection rates too may vary non-monotonically - initially increasing and then declining - in contagiousness.

In our basic model individuals are homogenous. We then turn to a society with two communities that differ in their returns from interaction - High and Low. Individuals in isolated communities exhibit different behavior: the High community has a higher rate of protection and interaction. As communities integrate, protection and interaction further increase in the High community while they fall in the Low community. Integration thus leads to falling (rising) infection in the High (Low) community.

The theoretical prediction on the relation between returns and equilibrium behavior is broadly consistent with empirical observation. Wellings et al. (1994) report that single people have more partners and are much more likely to use condoms as compared to cohabiting couples. Philipson and Posner (1993) report a negative correlation between education/income and HIV infection: they surmise that higher income raises the returns from the future and thereby leads to greater investments in protection (the use of condoms). This in turn lowers the rate of infection.

Our model and its predictions are also related to cybersecurity. ${ }^{3}$ The equilibrium property

\footnotetext{
${ }^{2}$ For a classical exposition of the theory of epidemiology, see Anderson and May (1991). For a recent survey on epidemics, see Gersovitz (2011).

${ }^{3}$ Estimates of the costs of cyber crime vary greatly. A recent study estimates the costs to be in the range of 300 billion USD to 1 trillion USD; this is between $0.4 \%$ and $1.4 \%$ of global GDP. A recent study for the UK Cabinet Office reported that the cost to the UK economy is over 27 billion USD per annum (Detica, (2011)). In 2009, roughly 10 million computers were infected with malware designed to steal online credentials. The
} 
of positive correlation between protection and interaction is consistent with the findings of Anderson et al. (2007) and Moore, Clayton and Anderson (2011) on the positive relation between investments in security and Internet use. The model predicts that the High community will have higher protection and interaction: this is consistent with the fact that larger firms are more active in securing themselves as compared to smaller firms (Anderson et al (2007)).

Our paper is a contribution to the economic study of epidemics and cybersecurity. It is useful to separate the existing research in economic epidemiology into two strands. The first strand of work takes interaction as given and explores the response in protection rates. This work includes Brito, Sheshinski and Intriligator (1991), Geoffard and Philipson (1996, 1997), Francis (1997), Goldman and Lightwood (2002), Gersovitz and Hammer (2004), Galeotti and Rogers (2013), and Chen and Toxvaerd (2014). A second (and complementary) group of papers assumes that protection is absent and studies the response in interaction. This work includes Philipson and Posner (1993) and Kremer (1996). To the best of our knowledge, the present paper is the first attempt to provide a unified treatment of interaction and protection. The analysis yields a number of new insights; we highlight two of them via a comparison with the benchmark models.

Compared to the 'pure protection' benchmark, our model yields lower rates of protection. This is because part of the population foregoes protection and responds instead by adapting interaction. But compared to that benchmark, infection rates are higher in our model. This tells us that differences in protection are 'insufficiently' compensated for by restricted interaction.

Consider next the 'pure interaction' benchmark, where protection is unavailable. The more a susceptible interacts, the greater the chances that he becomes infected and, in turn, transmits the disease to others around him. 'Pure interaction' models are thus characterized annual damages caused by malware is of the order of 9.3 billion Euros in Europe, while in the US the annual costs of identity theft are estimated at 2.8 billion USD (Moore, Clayton and Anderson (2009)). 
by the property that increasing returns from interaction raises infection (Kremer (1996)). In our setting, on the other hand, the more individuals value interaction the less inclined they are to respond to an epidemic by reducing interaction. This implies that higher returns from interaction lead to higher protection rates and - in sharp contrast to the 'pure interaction' benchmark - to lower infection.

Our results have potential policy implications. A first order implication is that demand for protection will be lower in a model where interaction levels are a choice variable. ${ }^{4}$ An important insight from the economic models of epidemiology is the externality in individual protection. In our model, choosing protection creates an additional externality: protected individuals interact more and this alters the pool of contacts. We show that this expands the scope for policy intervention, as compared to the 'pure protection' benchmark. Finally, our work suggests that subsidies on protection should target those valuing social interaction least, as doing so minimizes crowding-out effects.

The problem of computer network security has been extensively studied in electrical engineering and computer science; for an overview of this work see Alpcan and Basar (2011) and Anderson (2011). Aspnes, Chang and Yampolskiy (2006) (and the literature that follows them) study protection choices by nodes faced with a viral infection that spreads through a given network. Our paper contributes to this literature by proposing a general framework in which interaction (network) and security investments are both endogenous.

The rest of the paper is organized as follows. The basic model is presented in Section 2, and analyzed in Section 3. Section 4 studies heterogeneity. Section 5 concludes. All proofs are presented in the Appendix.

\footnotetext{
${ }^{4}$ The experience with swine flu vaccines is worth mentioning in this regard. Most OECD countries have large stocks of swine flu vaccines; for instance, in England, the NHS stock is estimated to have around 40 million vaccines in stock. This large stock of vaccines has provoked much discussion in recent years, see e.g., Times (2010). Our theoretical result points to one relatively unexplored reason for this large stock: lowered international travel and interaction in response to public measures on quarantine and the fears of epidemic.
} 


\section{Model}

The basic model has the following features. A continuum of individuals live for two periods. Each period, the agents individually decide how much social interaction to have (in a bar say, or in on-line activity). Social interaction is beneficial, but has drawbacks: interaction in the first period raises chances of a contagious infection, which reduces payoffs in the second period. To mitigate the chances of infection, each agent faces two options. He may reduce interaction in the first period, or invest in protection. The protection rate, the profile of interaction, and the contagiousness together determine the fraction of the population who become infected. We next lay out the details and notation of this model.

Social interaction. A typical agent is labeled $i$. We let $k_{i t} \geq 0$ denote the socialization 'effort' of agent $i$ in period $t=1,2$ : each period, $i$ selects $k_{i t}$ individuals uniformly at random from the pool of available contacts. ${ }^{5}$ We borrow this interpretation from Kremer (1996). ${ }^{6}$ Given an arbitrary subset $A$ of individuals, the probability that a new contact of $i$ is with an individual in $A$ is

$$
\mathbb{P}_{t}(\text { select contact in } A)=\frac{\int_{j \in A} k_{j t} d j}{\int_{j \in[0,1]} k_{j t} d j}
$$

Infection. There are two ways to become infected. An individual may become infected exogenously, with probability $\epsilon>0$. Or an individual may contract the disease by contagion. We let $\alpha$ denote the contagiousness of the disease, i.e. the probability of contracting the disease from interacting with an infected individual.

\footnotetext{
${ }^{5}$ The assumption on uniform selection is relaxed in Section 4, when considering heterogenous populations. There, we allow for 'search' to be directed.

${ }^{6}$ As in Kremer (1996), we abstract from strategic complementarity in social interactions. Making strategic complementarities more explicit and significant would introduce the possibility of multiple equilibria. While this would enrich the analysis, our thought is that the key trade-offs we identify would remain important in this richer framework.
} 
It is useful to define the incidence $q_{i t}$ facing agent $i$ in period $t$, indicating the probability that a new meeting of $i$ is with an infected individual. The total probability that (unprotected) individual $i$ becomes infected during period $t$ is then

$$
p_{i t}=\epsilon+(1-\epsilon)\left(1-\left(1-\alpha q_{i t}\right)^{k_{i t}}\right)
$$

Throughout, we assume that $\alpha$ is small so that $p_{i t}$ is well approximated by

$$
p_{i t}=\epsilon+(1-\epsilon) \alpha q_{i t} k_{i t} .
$$

Protection. Each individual faces the option to invest in protection, for a cost $\gamma>0 .^{7}$ The cost may be financial (as in the purchase of a condom, a vaccine or a computer security software) or reflect direct disutility (as in the case of condoms, or possible side-effects in the case of a vaccine). The binary variable $v_{i t}$ records the protection status of individual $i$ in period $t: v_{i t}=1$ if individual $i$ protects, and $v_{i t}=0$ otherwise. Protection perfectly immunizes an individual, so $p_{i t}=0$ if $v_{i t}=1 .{ }^{8}$ We will say that an individual is unprotected or susceptible if $v_{i t}=0$. The protection rate $V_{t}$ denotes the fraction of the population investing in protection. We let $I_{t}$ denote the infection rate, i.e. the fraction of individuals who become infected during period $t$ :

$$
I_{t}:=\int_{i \in[0,1]}\left(1-v_{i t}\right) p_{i t} d i .
$$

\footnotetext{
${ }^{7}$ We have opted for a simple formulation of protection: the costs are invariant with respect to level of interaction. This is a good model for a vaccine and for computer security software, but appears to be inappropriate for condoms where protection costs vary with frequency of interaction. We note that our main results continue to hold with variable costs of protection so long as the choice for protection is made ex-ante and applies to all interactions.

${ }^{8} \mathrm{We}$ assume that protection is perfect, i.e. an individual who invests in protection never contracts the disease. We note that (i) this assumption is justified if the probability of becoming infected is sufficiently reduced by protection and (ii) our results are unchanged if there is a small probability of getting the disease from the vaccine itself.
} 
Returns from interaction. We let $r_{i}\left(k_{i t}\right) \geq 0$ denote the returns from interaction for individual $i$ interacting according to $k_{i t}$ during period $t$. These are defined by

$$
r_{i}\left(k_{i t}\right):=\theta_{i} g\left(k_{i t}\right),
$$

where $\theta_{i}$ denotes the type of individual $i$, and $g$ is a twice continuously differentiable strictly concave function maximized at $\bar{k}>0$. Higher $\theta_{i}$ may reflect greater returns from interaction due to differences in human, physical or financial capital. A population is (ex ante) homogenous if all individuals have the same type, i.e. $\theta_{i}=\theta$ for all $i$, and heterogenous otherwise. Section 3 studies homogenous populations; Section 4 takes up the case of heterogenous populations.

Timing. The timing of the game is as follows. Each agent decides, when a period begins, whether or not to invest in protection and how much to interact during that period. During period $t$, unprotected agents become infected according to (3). An agent who becomes infected during period $t$ 'dies' when the period ends. The game ends after period 2, where all agents die.

Strategies and payoffs. A strategy for individual $i$ determines protection and interaction in each period before death. We assume that agents maximize expected lifetime returns from interaction, net of protection costs. ${ }^{9}$

\footnotetext{
${ }^{9} \mathrm{~A}$ simple way to account for risk aversion would involve subtracting $c p_{i 1}\left(1-p_{i 1}\right)$ to the RHS of equation (6) giving a susceptible's expected payoff, $c$ representing the cost of uncertainty for a susceptible. All results and proofs in this paper apply mutatis mutandis under this alternative specification. We are grateful to a referee for drawing our attention to this point.
} 
Equilibrium. The structure of the game as well as the distribution of types in the population are common knowledge. The model defines a game of complete information between individuals. We study the Nash equilibria of this game.

\section{Analysis}

This section presents our analysis of the choice of adaptive interaction and protection and its implications for infection in homogenous populations. We establish existence of a unique equilibrium, and compare its properties with those obtained in 'pure interaction' models on the one hand and 'pure protection' models on the other. We explore the effects of varying the contagiousness of the disease, and the returns from interaction.

We begin with some preliminary observations on the framework defined earlier allowing us to simplify the formal analysis of our model. Since all agents die when the final period ends, they need not be concerned about infection during that period. This yields $v_{i 2}=0$ and $k_{i 2}=\bar{k}$, for all $i$, in any equilibrium. An unprotected individual $i$ 's expected payoff may thus be simplified to

$$
\Pi_{i}\left(k_{i 1}, v_{i 1}=0\right)=r_{i}\left(k_{i 1}\right)+\left(1-p_{i 1}\right) r_{i}(\bar{k}),
$$

with $p_{i 1}$ given by (3): interacting according to $k_{i 1}$ in period 1 confers returns $r_{i}\left(k_{i 1}\right)$ during that period, but also reduces chances of a payoff in the period which follows.

Observe similarly that a protected individual $i$ 's expected payoff must in any equilibrium be given by

$$
\Pi_{i}\left(v_{i 1}=1\right)=2 r_{i}(\bar{k})-\gamma .
$$

Equations (6) and (7) allow us to reduce the initial setup to an equivalent but simpler one-shot game, and to focus exclusively on first-period variables. Henceforth time subscripts 
will be dropped, under the convention that a variable without a time subscript always refers to the first period. We will say that an equilibrium is interior if the protection rate $V$ lies in the open interval $(0,1)$.

Our first result establishes existence and uniqueness of an equilibrium in a homogenous population.

Proposition 1 Consider a homogenous population. An equilibrium exists and is unique.

We briefly describe the ways in which interaction and protection shape the payoffs of a susceptible. The negative externality exerted by unprotected individuals is governed by the incidence of the disease $q$ - the probability that an individual with whom an individual interacts happens to be infected. The incidence of the disease can be written as the product of two terms: (i) the probability $z$ of meeting another susceptible, and (ii) the probability $p$ that a susceptible is in fact infected.

Since in a homogenous population the incidence faced by all individuals is the same, and payoffs are strictly concave in interaction, then all suscpetibles must in any equilibrium choose the same interaction. Let here $k_{u}$ denote the interaction of unprotected individuals in a given equilibrium. Since $\bar{k}$ maximizes protected individuals' payoffs then, using (1) where $A$ now refers to the subset of susceptibles, the probability $z$ of meeting an unprotected individual is

$$
z=\frac{k_{u}(1-V)}{k_{u}(1-V)+\bar{k} V} .
$$

Substituting $q=z p$ in (3) and solving for $p$ yields

$$
p=\frac{\epsilon}{1-(1-\epsilon) \alpha z k_{u}} .
$$

A pair $\left(V, k_{u}\right)$ constitutes an interior equilibrium if and only if protected and unprotected 
individuals have equal payoffs, and

$$
k_{u}=\arg \max _{k}[r(k)+(1-\epsilon)(1-\alpha z p k) r(\bar{k})]
$$

with $z$ and $p$ satisfying, respectively, equations (8) and (9).

The proof of the Proposition has two steps. We first consider the pure interaction game given a fixed protection rate $V$ - this is a game played among unprotected individuals choosing how much to interact when the remaining fraction $V$ of the population protects. We show that this is a game of strategic substitutes, with a unique equilibrium. The 'equilibrium' payoffs attained in the pure interaction game are strictly increasing and continuous in the protection rate $V$. As payoffs from protection are fixed, existence and uniqueness of equilibrium in the overall game of protection and interaction follows.

The dual response to infection (restricted interaction and protection) plays a central role in our model. To illustrate its role, we now compare our equilibrium (a) with equilibrium in the 'pure protection' model with interaction fixed exogenously at the no-disease optimum $\bar{k}$ (benchmark 1) and (b) with equilibrium in the 'pure interaction' model with no protection (benchmark 2). We consider here the contrast between our model and benchmark model 1; the contrast with benchmark model 2 is developed after the statement of Proposition 3 below.

An important insight from the economic study of epidemiology is that a part of the benefits from protection comes from the reduced risk of infecting others, which agents fail to internalize. In our model, choosing protection creates an additional externality compared to the benchmark model 1: protected individuals interact more and this alters the pool of contacts. This remark suggests that the scope for policy intervention may be greater when individuals can adapt social interaction, as compared to the 'pure protection' benchmark . Our next result illustrates this point in two ways. First, we show that public protection programs may prove superfluous in benchmark 1, and yet be socially desirable in our framework of adaptive interaction. Second, 
we show that adaptive interaction ultimately induces higher rates of infection.

Proposition 2 Consider a homogenous population. Let $\left(V^{*}, I^{*}\right)$ and $\left(V^{1}, I^{1}\right)$ denote the equilibrium protection and infection rates in our model and benchmark model 1, respectively. Then:

1. $V^{*} \leq V^{1}$, with strict inequality if $V^{*}$ and $V^{1} \in(0,1)$.

2. $V^{*}$ and $V^{1} \in(0,1) \Rightarrow I^{*}>I^{1}$.

The proof of the first part builds on the observation that, for any given rate of protection, the resulting incidence of the disease is higher with fixed interaction as compared to the case where unprotected individuals adapt (and hence lower) interaction. This pushes the returns from protection upwards, inducing higher protection rates. The second part is more delicate: protection is higher in the benchmark model, but adaptive interaction in our model may compensate for the difference in protection rates. Our proof shows that while interaction adapts downward, it does so 'insufficiently'. As a result, incidence and infection are both higher in our model, as compared to the benchmark model 1.

Consider next the implications of increasing returns from interaction. In what follows $V(\theta)$ denotes the equilibrium protection rate in a homogeneous population with type $\theta$.

Proposition 3 Suppose $V(\theta) \in(0,1)$. An increase in the returns $\theta$ induces a first-order stochastic dominance shift in the equilibrium profile of interaction and raises equilibrium protection. Moreover, depending on the function $g$, increasing $\theta$ may reduce equilibrium infection rates.

The intuition behind the first part of Proposition 3 is straightforward. Higher returns from interaction enhance the appeal of protection relative to the option of reducing interaction. In equilibrium, more agents protect. This lowers the incidence $q$ of the disease, allowing in turn susceptibles to interact more. 
The second part of the Proposition is perhaps most interesting. Increased protection tends to lower infection; yet higher interaction from the susceptible part of the population pushes infection up. This raises the question: how is infection globally affected by increased returns from interaction? The key issue is the elasticity of interaction: if this elasticity is not too high, then the effect of higher protection will prevail. We show in the proof, using an example with logarithmic returns, that infection rates may fall with a rise in $\theta$.

The latter observations are helpful in bringing out the differences between our model and the 'pure interaction' benchmark model 2. In the absence of protection, the more individuals value interaction, the greater the negative equilibrium externality each agent imposes on others. In the 'pure interaction' benchmark, therefore, an increase in $\theta$ unambiguously raises the infection rate (Kremer (1996)). As shown in the discussion above, in our model the effects of increasing returns $\theta$ are very different. The more individuals value interaction, the less inclined they are to respond to an epidemic by reducing interaction: this pushes protection up and leads to falling infection rates for a broad class of applications.

We turn finally to the effects of contagiousness on behavior and infection rates. Our analysis establishes that there are two phases in the equilibrium response to contagiousness. In phase I, at low $\alpha$, the response to higher contagiousness is entirely through reduced interaction. In phase II, at high $\alpha$, greater contagiousness induces more protection but there is no reduction of social interaction. Social interaction among the unprotected stays constant, but because more individuals protect (and protected individuals interact more) the profile of interaction undergoes a first-order stochastic dominance shift. Hence, somewhat surprisingly, social interaction is non-monotonic in contagiousness: it first decreases but eventually rises.

Proposition 4 Consider a homogenous population. Equilibrium protection $V$ increases with contagiousness $\alpha$. Moreover, there exist $\underline{\alpha}$ and $\bar{\alpha}, \underline{\alpha} \leq \bar{\alpha}$, such that: ${ }^{10}$

\footnotetext{
${ }^{10}$ Lemma 3 in the Appendix shows that both threshold values are decreasing in $\epsilon$, the exogenous probability of infection.
} 
1. If $\alpha \leq \underline{\alpha}$ then a reduction in $\alpha$ induces a first-order stochastic dominance shift in the equilibrium profile of interaction. Infection rate changes depend on the elasticity of interaction with respect to $\alpha$.

2. If $\underline{\alpha}<\alpha<\bar{\alpha}$ then an increase in $\alpha$ induces a first-order stochastic dominance shift in the equilibrium profile of interaction, and a decrease of the infection rate $I$.

3. If $\alpha \geq \bar{\alpha}$ then all agents protect, and $I=0$.

We start by observing that if $V=0$ then the negative externality exerted by unprotected individuals is strictly increasing in contagiousness, $\alpha$. This implies that the equilibrium interaction of unprotected individuals, $k_{u}$, decreases in $\alpha$ and completes the arguments for part 1.

Consider next an interior equilibrium: an individual must be indifferent between protecting or not. Since payoffs from protection are unaffected by $\alpha$, the payoffs earned by susceptibles must be unchanged as well. This implies that the equilibrium externality $\alpha q(\alpha)$ is constant in $\alpha$. As $\alpha$ is rising, the incidence $q($.$) must be falling. Now consider protection. Suppose$ that protection were falling in $\alpha$. Then the only way to reduce the incidence $q(\alpha)$ would be to reduce the interaction $k_{u}$. But we have already proven that the externality $\alpha q(\alpha)$ is constant in $\alpha$, and so is $k_{u}$. This contradiction establishes that protection rates must be increasing in $\alpha$. As the equilibrium $k_{u}$ is constant in $\alpha$, and the fraction of protected individuals rises, an increase in contagiousness $\alpha$ thus induces a first-order stochastic dominance shift in the equilibrium profile of interaction. This completes the argument for part 2.

We conclude with a brief discussion on the effect of contagiousness on infection rates. Kremer (1996) observes that (in the absence of protection) higher contagiousness may lead to lower infection rates if the elasticity of interaction with respect to contagiousness is greater than one. The remark on infection rates in the first part of Proposition 4 follows similar logic. 
The contrast is, however, sharp if we look at infection rates in an interior equilibrium (i.e. $\alpha \in(\underline{\alpha}, \bar{\alpha}))$ : in this case higher contagiousness always lowers infection rates. The idea is as follows. The externality $\alpha q(\alpha)$ remains constant over this range. The probability of infection of an unprotected individual must therefore remain unchanged as well. But $I=(1-V) p$, so the infection rate $I$ falls, since equilibrium $V$ is rising in $\alpha$.

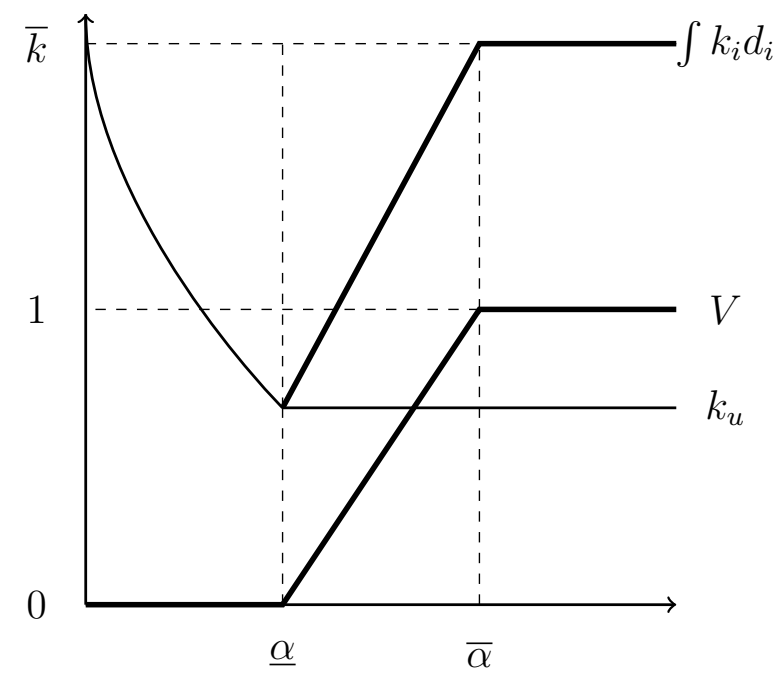

Figure 1

Contagiousness $\alpha$ is measured on the $\mathrm{x}$-axis. We measure on the $\mathrm{y}$-axis the equilibrium average interaction in the population, the equilibrium vaccination rate $V$, and the equilibrium interaction of unprotected agents $k_{u}$.

\section{Heterogenous populations}

This section studies the effect of population heterogeneity on behavior and infection rates. We model heterogeneity in terms of differences in returns from interaction. Our goal is to examine the interplay between this heterogeneity and the choice between restricted interaction and protection. Observe that the case where the different 'types' of individuals are completely 
segregated is covered by Proposition 3 above. Our analysis reveals that the integration level between different types has powerful effects on behavior and infection rates.

We shall suppose that a heterogenous population consists of two equal size communities of individuals with types $\theta_{H}$ and $\theta_{L}$ respectively, $\theta_{H}>\theta_{L}$. A parameter $\beta$ measures integration between the two communities. With probability $\beta$ (resp., $1-\beta$ ), social interaction takes place across communities (resp., within one's own community). Thus, for $\beta=0$ the two communities of individuals are completely segregated, while for $\beta=1$ all interaction takes place across communities. ${ }^{11}$ The probability that a new meeting of an individual in community $J$ is with an individual in subset $A \subset J^{\prime}$ is thus

$$
\mathbb{P}\left(i \in J \text { selects contact in } A \subset J^{\prime}\right)=\left((1-\beta) I_{\left\{J=J^{\prime}\right\}}+\beta I_{\left\{J \neq J^{\prime}\right\}}\right) \frac{\int_{i \in A} k_{i} d i}{\int_{i \in J^{\prime}} k_{i} d i} \text {, }
$$

where as usual $I_{\{.\}}$denotes an indicator variable.

We first study the effects from integrating communities moderately. We then explore, through an example, the multiplicity of equilibria arising when most interaction takes place across communities. Throughout, we focus the analysis on the more interesting case where the equilibrium protection rate in a homogenous population with type $\theta_{J}$ is interior, i.e. $V\left(\theta_{J}\right) \in(0,1), J \in\{H, L\} .^{12}$

Consider first $\beta=0$. When $\beta=0$, results from Proposition 3, concerning homogenous populations, apply. Equilibrium is unique and entails strictly higher incidence in community $L$ than in community $H$. So consider now a small increase in $\beta$. Other things equal, an

\footnotetext{
${ }^{11}$ For consistency, and to abstract from issues regarding community size, we assume that if interaction in community $J$ is greater than it is in community $J^{\prime}$ then only a fraction of individuals in community $J$ interact across communities. This ensures in a simple way that total interaction from community $J$ to $J^{\prime}$ equals total interaction from $J^{\prime}$ to $J$. Formally, if $\int_{i \in J} k_{i} d i>\int_{i \in J^{\prime}} k_{i} d i$ then partition community $J$ as $J_{1} \bigcup J_{2}$ such that $\int_{i \in J_{1}} k_{i} d i=\int_{i \in J^{\prime}} k_{i} d i$, and individuals in $J_{2}$ interact within $J_{2}$ only.

${ }^{12}$ The analysis of the other cases is similar, but the results are plainer. For instance if $V\left(\theta_{H}\right)=V\left(\theta_{L}\right)=0$ then for any $\beta$, equilibrium is unique and entails all individuals in both communities remaining unprotected. Similarly, if $V\left(\theta_{H}\right)=V\left(\theta_{L}\right)=1$ then for any $\beta$, equilibrium is unique and entails all individuals in both communities protecting.
} 
individual in community $H$ faces higher incidence compared to $\beta=0$, since he will interact with some probability with an individual from community $L$ where the incidence is higher. This raises incentives to protect in community $H$. By symmetry, an individual in community $L$ now faces lower incidence since he will interact, with some probability, with an individual in community $H$ where the incidence is lower. This lowers incentives to protect in community $L$. Integrating communities moderately therefore benefits $L$ types: As integration grows, the burden of protection tends to fall on $H$ types, while $L$ types free-ride. The next proposition summarizes these insights, and explores their implications for interaction and infection in the two communities.

Proposition 5 Consider a heterogenous population such that equilibrium is interior in both isolated communities, so that $V\left(\theta_{J}\right) \in(0,1), J \in\{H, L\}$. Then an equilibrium exists for all levels of integration $\beta$, and is unique over a range $[0, \bar{\beta}]$, where $\bar{\beta} \in(0,1 / 2)$. Moreover, over this range, increasing $\beta$ induces (i) higher (lower) protection in community $H$ (in community L), (ii) lower (higher) infection in community $H$ (in community L) and (iii) a (inverse) first-order stochastic dominance shift in the equilibrium profile of interaction of community $H$ (community L).

We explained part (i) above. We now develop the intuition underlying parts (ii) and (iii). By continuity, for low values of $\beta$ the unique equilibrium is interior in both communities. So, for small values of $\beta$, individuals in each community must remain indifferent between protection and no protection. Equilibrium payoffs of unprotected individuals therefore remain unchanged as $\beta$ varies, given that payoffs of protected individuals are constant. Since payoffs of unprotected individuals are strictly falling in the incidence of the disease, this implies that the incidence faced by individuals in both communities must remain unaffected by changes in $\beta$. This in turn implies that, in each community, susceptible interaction must be unchanged, and so must their probability of infection. However, since the protection rate grows (falls) in 
$H(L)$, the fraction of infected individuals must in fact decrease (increase) in community $H$ $(L)$. Finally, the arguments above establish that more integration induces higher protection in community $H$ but no reduction of interaction by unprotected individuals in that community. Hence, an increase in $\beta$ induces a first-order stochastic dominance shift in the equilibrium profile of interaction of community $H$. Similar reasoning establishes the claim for community $L$.

There are interesting implications of Proposition 5. To illustrate, suppose that prior to the stage at which individuals make a decision a social planner is able to allocate protection to a small subset of the population. If communities are largely segregated ( $\beta$ small), the unique equilibrium is interior in both communities. Any allocation of the social planner therefore induces complete crowding-out of private incentives, leaving the resulting equilibrium unaffected. Suppose, on the other hand, that communities are sufficiently integrated so that in the unique equilibrium all individuals in community $H$ protect while all individuals in community $L$ remain unprotected. In this case, allocating protection to high types once again crowds-out private incentives, whereas allocating protection to low types strictly improves welfare in the resulting equilibrium, due to the positive externalities from protection within community $L$. More generally, targeting individuals valuing interaction least tends to minimize crowding-out in our model and as such, somewhat counter-intuitively, makes it attractive for public policy to protect individuals valuing social interaction least.

We have so far focused on the case of limited integration between communities. Greater integration typically implies multiple equilibria. To bring out this point in the simplest way, we next consider an example where $\beta=1$ and focus on a range of parameters where isolated communities exhibit interior equilibria. The case $\beta=1$ corresponds, e.g., to a situation where all sex is heterosexual. We show in the example that three equilibria exist. The first equilibrium is interior in both communities. The other two equilibria are extremal: all individuals 
protect in one of the two communities, while all individuals remain unprotected in the other. In these equilibria, a protected individual interacts only with unprotected individuals, and vice versa. Protection is thus attractive for those who do protect, while remaining unprotected is optimal under the guarantee never to meet an infected individual. In this setting, it is possible to show that social welfare is highest in the corner equilibrium where $H$ types protect and $L$ types do not. Extremal equilibria are attractive since they maximize meetings between protected and unprotected individuals. Of the two extremal equilibria, that in which high types protect dominates due to the fact that the loss from reduced interaction is worse for high types than low.

Proposition 6 Consider a heterogenous population, $\beta=1$, and $q\left(\theta_{J}\right) \in(0, \epsilon), J \in\{H, L\}$. There exist exactly three equilibria. In equilibrium 1 all individuals in groups $H$ protect and all individuals in group $L$ remain unprotected. In equilibrium 2 all individuals in group $L$ protect and all individuals in group $H$ remain unprotected. Equilibrium 3 is interior in both groups. If $W_{k}$ denotes average social welfare in these equilibria then $W_{1}>W_{2}>W_{3}$.

\section{Conclusion}

Social and economic interactions generate rewards but also facilitate the spread of infections. Individuals can respond to this risk by restricting interaction and by investing in protection. This paper develops a model that examines the trade-off between these two actions and the implications for the prevalence of infections.

We study a setting in which an infection may be contracted either exogenously or through

contact with an infected individual. Individuals decide on how much to interact with others and whether to protect themselves. We study the equilibrium levels of protection and interaction and the population rate of infection. 
We establish that there exists a unique equilibrium. We then derive a number of interesting implications of the co-determination of interaction and protection. There is a threshold property with regard to the effects of contagiousness. Below a threshold level, interaction falls in contagiousness, while it rises above the threshold. Infection rates too may move nonmonotonically: below a threshold level they rise in contagiousness and above the threshold they fall with contagiousness.

In the basic model, all individuals are homogenous. We extend the model to study a society composed of two communities that differ in their returns from interaction - High and Low. Individuals in isolated communities exhibit different behavior: the High community has a higher rate of protection and interaction. As communities integrate, protection and interaction further increase in the High community while they fall in the Low community. Integration thus leads to lower (higher) disease prevalence in the High (Low) community.

\section{Appendix}

Remark: We focus throughout this Appendix on the 'reduced' one-shot game derived in the beginning of Section 3 with payoffs given by (6) and (7), and thus drop the time subscripts from all notation.

Consider a homogenous population. Define the pure interaction game given protection rate $V$ (PIGGV) as the game played among unprotected individuals choosing how much to interact when the remaining fraction $V$ of the population protects. Let $q_{i}\left(k_{u} ; V\right)$ denote the incidence of the disease facing individual $i$ in the PIGGV, when other unprotected individuals interact according to $k_{u}$. Note that $q_{i}\left(k_{u} ; V\right)=p_{-i} . z_{i}$, where $z_{i}$ is the probability that an arbitrary meeting of $i$ is with an unprotected individual and $p_{-i}$ is the probability that an unprotected individual other than $i$ is in fact infected. Since the population is composed of a 
continuum of individuals, notice that $p_{-i}=p$ and $z_{i}=z$, with $z$ given by (8). Moreover, using (3) yields $p=\epsilon+(1-\epsilon) \alpha p z k_{u}$, and then (9) upon rearrangement. This shows, in particular, that $q_{i}\left(k_{u} ; V\right)=p . z$ strictly increases in $k_{u}$ ( $z$ increases in $k_{u}$, while $p$ is increasing in each of $z$ and $\left.k_{u}\right)$.

The payoffs of $i$ in the PIGGV as a function of $k_{i}$ as well as other players' actions $k_{-i}$ can be written

$$
\Pi_{i}\left(k_{i}, k_{-i}\right)=r_{i}\left(k_{i}\right)+\left(1-p_{i}\left(k_{i}, q_{i}\left(k_{-i} ; V\right)\right)\right) r_{i}(\bar{k}),
$$

where $p_{i}\left(k_{i}, q_{i}\left(k_{-i} ; V\right)\right)=\epsilon+(1-\epsilon) \alpha q\left(k_{-i} ; V\right) k_{i}$. Taking cross derivatives yields

$$
\frac{\partial \Pi_{i}}{\partial k_{i} \partial k_{-i}}=-\alpha(1-\epsilon) \frac{\partial q_{i}}{\partial k_{-i}} r_{i}(\bar{k})>0,
$$

where the inequality follows from the arguments above. The PIGGV is thus a game of strategic substitutes. Standard considerations then establish that an equilibrium of the PIGGV exists, is unique, and symmetric (uniqueness and symmetry follow the fact that the incidence of the disease is the same for all individuals, together with the strict concavity of payoffs with respect to interaction). Let in what follows $\Pi(V)$ denote the equilibrium payoff achieved by unprotected individuals in the PIGGV. The next lemma is useful:

Lemma $1 \Pi(V)$ is continuously differentiable.

Proof: First we show that $k_{u}(V)$ - the equilibrium interaction of unprotected individuals in the PIGGV - is continuously differentiable. Using first order conditions yields a continuously differentiable $G(.,$.$) implicitly defining k_{u}(V)$ through the equation $G\left(k_{u}(V), V\right)=k_{u}(V)$. Hence, using the Implicit Function Theorem shows that $k_{u}(V)$ is continuously differentiable. Next, note that $q_{i}\left(k_{u} ; V\right)$ (the incidence of the disease facing individual $i$ in the PIGGV, when other unprotected individuals interact according to $k_{u}$ ) is continuously differentiable. 
The equilibrium value of the incidence of the disease in the PIGGV, $q(V)$, is thus continuously differentiable too since $q(V)=q_{i}\left(k_{u}(V) ; V\right)$.

The proof is concluded by noting that the probability of infection is continuously differentiable in the incidence $q$, while the returns from interaction are continuously differentiable in $k_{u}$.

Proof of Proposition 1: Our proof uses the analysis of the PIGGV given above. Recall that $\Pi(V)$ denotes the equilibrium payoff achieved by unprotected individuals in the PIGGV. By investing in protection, an individual achieves payoff $2 r(\bar{k})-\gamma$. Thus, an equilibrium of the overall game with protection rate $V$ and in which unprotected individuals interact according to $k_{u}$ exists if and only if $k_{u}$ is the equilibrium interaction of unprotected individuals in the PIGGV and:

1. if $V=0$ then $\Pi(0)>2 r(\bar{k})-\gamma$;

2. if $V=1$ then $\Pi(1)<2 r(\bar{k})-\gamma$;

3. if $V \in(0,1)$ then $\Pi(V)=2 r(\bar{k})-\gamma$.

Next, we claim that $\Pi(V)$ is strictly increasing in $V$. To establish the claim, we first show that $q(V)$ - the equilibrium value of the incidence of the disease in the PIGGV - strictly decreases in $V$. Suppose, for a contradiction, that $q(V)$ increases in $V$. Each unprotected individual would then find it in his best interest to interact (weakly) less following a rise in $V$, i.e. $k_{u}$ must decrease in $V$. Since $z=k_{u}(1-V) /\left(k_{u}(1-V)+\bar{k} V\right)$, this also implies that $z$ must strictly decrease in $V$ (more individuals protect, and unprotected individuals interact less). But then, by (9) (giving $p$ as a function of $z$ and $k_{u}$ ), each unprotected individual must in equilibrium exhibit strictly lower probability of infection following a rise in $V$. We have thus established that if $q(V)$ increases in $V$, then both $z$ and $p$ must strictly decrease in $V$. 
But $q=z p$, so that $q(V)$ must strictly decrease in $V$ too - an obvious contradiction. Hence, $q(V)$ is strictly falling in $V$. The maximum payoff achieved by unprotected individuals in the PIGGV thus strictly increases in $V$. This establishes that $\Pi(V)$, the equilibrium payoff achieved by unprotected individuals in the PIGGV, strictly increases in $V$.

Combining the first part of the proof with the fact that $\Pi(V)$ strictly increases in $V$ establishes existence and uniqueness of an equilibrium. Specifically:

1. if $\Pi(0)>2 r(\bar{k})-\gamma$ then $V=0$ in the unique equilibrium;

2. if $\Pi(1)<2 r(\bar{k})-\gamma$ then $V=1$ in the unique equilibrium;

3. if $\Pi(0)<2 r(\bar{k})-\gamma<\Pi(1)$ then the equilibrium protection rate is uniquely determined by

$$
\Pi(V)=2 r(\bar{k})-\gamma
$$

Proof of Proposition 2: We begin with part 1. Let $\Pi^{1}(V)$ denote the payoffs of unprotected individuals with fixed interaction $\bar{k}$ when a fraction $V$ of the population protects. Fixing $V$, note that the probability $z$ of meeting an unprotected individual is strictly greater with fixed interaction than in the PIGGV. Since, by (9), $p$ is increasing in each of $z$ and $k_{u}$ this also implies that the probability of infection is strictly greater with fixed interaction than in the PIGGV, as is ultimately the incidence $q=z p$. This establishes that $\Pi(V)$, the maximum payoff achieved by unprotected individuals (in equilibrium) in the PIGGV, must be greater than $\Pi^{1}(V)$. That $V^{*} \leq V^{1}$, where $V^{*} / V^{1}$ denote the equilibrium protection rate under adaptive/fixed interaction, now follows immediately $\left(V^{*} / V^{1}\right.$ are uniquely determined by the intersection of the strictly increasing functions $\Pi(V) / \Pi^{1}(V)$ with the 'horizontal' schedule equal to $2 r(\bar{k})-\gamma)$. 
We now prove part 2 of the Proposition. $V^{*}$ and $V^{1} \in(0,1)$ implies that in both cases (fixed interaction and adaptive interaction) the equilibrium payoffs achieved by unprotected individuals equal $2 r(\bar{k})-\gamma$. Since for any given value of the incidence the option to adapt interaction raises the maximum payoffs attainable by unprotected individuals, we must have $q^{*}>q^{1}$. The proof is concluded by noting that

$$
\begin{aligned}
I^{*}-I^{1} & =p^{*}\left(1-V^{*}\right)-p^{1}\left(1-V^{1}\right) \\
& =p^{*}\left[\left(1-V^{*}\right)+z^{*}-z^{*}\right]-p^{1}\left[\left(1-V^{1}\right)+z^{1}-z^{1}\right] \\
& =\left[q^{*}-q^{1}\right]+p^{*}\left[\left(1-V^{*}\right)-z^{*}\right] \\
& >0 .
\end{aligned}
$$

The last equality follows from noting that $\left(1-V^{1}\right)=z^{1}$. The inequality is due to the remark above that $q^{*}>q^{1}$, and the observation that $\left(1-V^{*}\right)-z^{*}>0$.

Proof of Proposition 3: In this proof $k_{u}(\theta), q(\theta), V(\theta)$, and so on will be used to denote equilibrium values in a homogenous population of type $\theta$. The proof is in three steps. We first establish $d q(\theta) / d \theta<0$. We then show that $d V(\theta) / d \theta>0$. Finally, we show that with logarithmic returns then $d I(\theta) / d \theta<0$.

Step 1: Let $p\left(k_{i}, q_{i}\right)$ denote the infection probability of $i$ interacting according to $k_{i}$ and facing incidence $q_{i}$ (notice that, fixing $k_{i}$, the probability of infection is independent of $\theta_{i}$ ). Observe that $\partial p / \partial q_{i}>0$.

The equilibrium indifference condition between vaccinating or not can be written as

$$
2 \theta g(\bar{k})-\gamma=\theta g\left(k_{u}(\theta)\right)+\left(1-p\left(k_{u}(\theta), q(\theta)\right)\right) \theta g(\bar{k}) .
$$


Differentiating with respect to $\theta$ yields

$$
2 g(\bar{k})=g\left(k_{u}(\theta)\right)+\left(1-p\left(k_{u}(\theta), q(\theta)\right)\right) g(\bar{k})-\frac{\partial p}{\partial q} \frac{d q}{d \theta} \theta g(\bar{k})
$$

where we have used the fact that $k_{u}(\theta)$ is optimal for unprotected individuals, given $q(\theta)$. Rearranging yields

$$
\frac{d q}{d \theta}=-\frac{2 g(\bar{k})-g\left(k_{u}(\theta)\right)-\left(1-p\left(k_{u}(\theta), q(\theta)\right)\right) g(\bar{k})}{\frac{\partial p}{\partial q} \theta g(\bar{k})} .
$$

Step 1 is concluded by noting that both numerator and denominator in the expression above are strictly positive.

Step 2: First notice using (10) that the first step of the proof implies $d k_{u}(\theta) / d \theta>0$ (lower incidence reduces the marginal cost of interaction). Suppose now for a contradiction that $d V(\theta) / d \theta \leq 0$. Since $z=k_{u}(1-V) /\left(k_{u}(1-V)+\bar{k} V\right)$, we obtain $d z(\theta) / d \theta>0$. This in turn yields $d p(\theta) / d \theta>0$, where we have used (9). Finally, since $q=z p$, we obtain $d q(\theta) / d \theta>0$, contradicting step 1. This establishes $d V(\theta) / d \theta>0$.

Step 3: We have $I(\theta)=p(\theta)(1-V(\theta))$, where $p(\theta)=p\left(k_{u}(\theta), q(\theta)\right)$. Since by step 2 $d V(\theta) / d \theta>0$, then $d p(\theta) / d \theta \leq 0 \Rightarrow d I(\theta) / d \theta<0$. We will now show that with logarithmic returns the equilibrium probability of infection is non-increasing in $\theta$.

Fix $\bar{k}>0$ and consider $g(k)$ defined in the following way: ${ }^{13} g(k)=\ln k$ if $k \leq \bar{k}$, and $g(k)=\ln \bar{k}$ if $k>\bar{k}$. Straightforward computations establish $k_{u}(\theta) q(\theta)=(\alpha(1-\epsilon) \ln \bar{k})^{-1}$. The proof is concluded by nothing that $p(\theta)=\epsilon+(1-\epsilon) \alpha q(\theta) k_{u}(\theta)=\frac{\epsilon \ln \bar{k}+1}{\ln \bar{k}}$.

Proof of Proposition 4: Our proof exploits the impact of $\alpha$ on the equilibrium of the

\footnotetext{
${ }^{13}$ While the returns defined in this example violate the assumptions of the basic model ( $g$ is here neither twice continuously differentiable, nor strictly concave), simple inspection establishes that all our proofs and results extend in this case too.
} 
PIGGV. Specifically, we first show that a rise in $\alpha$ induces a rise in the equilibrium value of the externality $\alpha . q(V ; \alpha)$ in the PIGGV. We then go on to show that $\Pi(V ; \alpha)$, the equilibrium payoff achieved by unprotected individuals in the PIGGV, strictly decreases in the contagiousness $\alpha$. Finally we show that a (uniform) fall of the equilibrium payoff achieved by unprotected individuals in the PIGGV implies a rise in the equilibrium protection rate of the overall game.

Step 1: Let $\alpha_{2}>\alpha_{1}$, and fix $V$. Let $q(V ; \alpha)$ denote the equilibrium incidence in the PIGGV under contagiousness $\alpha$. Suppose for a contradiction that $\alpha_{2} . q\left(V ; \alpha_{2}\right) \leq \alpha_{1} . q\left(V ; \alpha_{1}\right)$. An unprotected individual would then find it optimal to interact at least as much under $\alpha_{2}$ as under $\alpha_{1}$ (due to lower marginal cost of interaction). Since $V$ is fixed, we must then have $z_{2} \geq z_{1}$. But then, from equation (9), each unprotected individual has higher probability of infection under $\alpha_{2}$. Given that $q=z p$, this implies $q\left(V ; \alpha_{2}\right) \geq q\left(V ; \alpha_{1}\right)$, and ultimately $\alpha_{2} \cdot q\left(V ; \alpha_{2}\right)>\alpha_{1} \cdot q\left(V ; \alpha_{1}\right)$ - contradicting our original assumption. Thus a rise in $\alpha$ induces a rise in the equilibrium externality $\alpha . q(V, \alpha)$ in the PIGGV.

Step 2: From step 1, the (PIGGV) equilibrium value of $\alpha . q(V ; \alpha)$ strictly increases in $\alpha$. In equilibrium, the maximum payoff achieved by unprotected individuals in the PIGGV thus strictly decreases in $\alpha$. This implies that $\Pi(V ; \alpha)$, the equilibrium payoff achieved by unprotected individuals in the PIGGV, strictly decreases in $\alpha$.

Step 3: It was shown in the proof of Proposition 1 that the equilibrium protection rate $V$ is determined by the intersection of (the strictly increasing function of $V) \Pi(V ; \alpha)$ and the horizontal schedule $2 r(\bar{k})-\gamma$. From step 2, we know that $\Pi(V, \alpha)$ is strictly decreasing in $\alpha$. It then follows that the equilibrium protection rate $V$ must increase in $\alpha$. Specifically, there exist $\underline{\alpha}$ and $\bar{\alpha}, \underline{\alpha} \leq \bar{\alpha}$, such that in equilibrium: $V=0$ if $\alpha \leq \underline{\alpha}, V \in(0,1)$ if $\alpha \in(\underline{\alpha}, \bar{\alpha})$, and $V=1$ if $\alpha \geq \bar{\alpha}$.

We can now conclude the proof of the proposition. Let $\underline{\alpha}$ and $\bar{\alpha}$ be as defined in step 
3. Consider first $\alpha \leq \underline{\alpha}$. The equilibrium protection rate is $V=0$. It follows that (in the overall game) the equilibrium value of the externality is $\alpha . q(0 ; \alpha)$. From step $1, \alpha . q(0 ; \alpha)$ strictly increases in $\alpha$. From the optimization problem (10), the equilibrium interaction $k_{u}$ of unprotected individuals thus strictly decreases in $\alpha$. Consider next $\alpha \in(\underline{\alpha}, \bar{\alpha})$. We have over this range $V \in(0,1)$; so individuals are indifferent between protecting or not. The maximum payoff attainable by unprotected individuals is thus $2 r(\bar{k})-\gamma$ over the entire range - the payoff they could achieve by protecting. Since the maximum payoff attainable by unprotected individuals is strictly decreasing in the externality $\alpha . q$, the equilibrium value of $\alpha . q$ must remain constant over the whole range $\alpha \in(\underline{\alpha}, \bar{\alpha})$. This in turn implies that over this range (i) the equilibrium incidence $q$ strictly decreases, (ii) the equilibrium interaction $k_{u}$ of unprotected individuals remains constant, and (iii) the equilibrium probability of infection $p$ remains constant too. Since $q=z p$, the equilibrium value of $z$ thus strictly falls over this range. The identity

$$
V=\frac{k_{u}(1-z)}{k_{u}+z\left(\bar{k}-k_{u}\right)}
$$

then shows that $V$ strictly rises over this range. Finally, for $\alpha \geq \bar{\alpha}$ all individuals protect in equilibrium, and each individual interacts according to $\bar{k}$.

Lemma 2 Let $\underline{\alpha}$ and $\bar{\alpha}$ as defined in Proposition 4. Then both variables are decreasing in $\epsilon$, the exogenous probability of infection.

Proof: It is easy to see that $\Pi(V)$, the equilibrium payoff of the PIGGV, decreases with $\epsilon$. This follows from the fact that the payoff of a susceptible may be written as $r_{i}\left(k_{i}\right)+(1-\epsilon)(1-$ $\left.\alpha q_{i} k_{i}\right) r_{i}(\bar{k})$ : best responses in the PIGGV are thus increasing in $\epsilon$. Intuitively, increasing $\epsilon$ make susceptibles 'fatalistic', lowering the marginal cost of interaction. By (9), therefore, the equilibrium probability of infection in the PIGGV increases in $\epsilon$. Using (6) then shows that 
$\Pi(V)$ decreases in $\epsilon$.

Recall that the equilibrium protection rate $V$ in the overall game is given by the intersection of $\Pi(V)$ (an increasing schedule) and $2 r(\bar{k})-\gamma$. Using the first step, the equilibrium $V$ thus increases in $\epsilon$. This concludes the proof since we know from Proposition 4 that $V$ increases in $\alpha$, and $\underline{\alpha}$ (resp. $\bar{\alpha})$ is defined as the lowest value (resp. highest value) for which $V>0$ (resp. $V<1)$.

Proof of Proposition 5: The proof of existence follows the line of Lemma 1, and is therefore omitted. We next show that an equilibrium is unique (and interior in both communities) for $\beta$ positive but small.

The equilibrium protection rates $V\left(\theta_{J}\right), J \in\{H, L\}$, are interior for types of homogenous populations. At $\beta=0$, the unique equilibrium is thus interior in both communities. Let $Q_{J}$ denote the incidence within community $J$, i.e.

$$
Q_{J}=\frac{\int_{i \in J}\left(1-v_{i}\right) p_{i} k_{i} d i}{\int_{i \in J} k_{i} d i} .
$$

Making $\beta$ and $Q_{-J}$ into parameters and focusing on community $J$ we can - by analogy with the analysis of homogenous populations - define within community $J$ the pure interaction game given protection rate $V_{J}$. This is a game played among unprotected individuals in community $J$ choosing how much to interact when the remaining fraction $V_{J}$ of community $J$ protects, a fraction $\beta$ of interaction occurs across communities, and the incidence of the disease in the other community is $Q_{-J}$. Let $\Pi\left(V_{J} ; \beta, Q_{-J}\right)$ denote the equilibrium payoff achieved by the players in this game; notice that $\Pi\left(V_{J} ; 0, Q_{-J}\right)=\Pi\left(V_{J}\right)$, where $\Pi\left(V_{J}\right)$ denotes the equilibrium payoff achieved by unprotected individuals in the PIGGV of Lemma 1. Repeating the same steps as in the proof of Lemma 1 establishes that $\Pi\left(V_{J} ; \beta, Q_{-J}\right)$ is strictly increasing in $V_{J}$. 
Taylor expanding yields

$$
\Pi\left(V_{J} ; \beta, Q_{-J}\right)=\Pi\left(V_{J} ; 0, Q_{-J}\right)+O(\beta)=\Pi\left(V_{J}\right)+O(\beta)
$$

The payoff to protected individual in community $J$ is $2 r_{J}(\bar{k})-\gamma \in(\Pi(0), \Pi(1)), J \in$ $\{H, L\}$, since the unique equilibrium in a homogenous population of type $\theta$ is interior for both types $\theta=\theta_{H}$ and $\theta=\theta_{L}$. This implies, by (12), that for $\beta$ small $2 r_{J}(\bar{k})-\gamma \in$ $\left(\Pi\left(0, \beta, Q_{-J}\right), \Pi\left(1, \beta, Q_{-J}\right)\right), J \in\{H, L\}$, and arbitrary $Q_{-J}$. Hence, for $\beta$ small any equilibrium of our game must be interior for both communitys (i.e. $V_{J} \in(0,1), J \in\{H, L\}$ ).

Lastly, we show that, for any $\beta$, at most one equilibrium exists that is interior for both communities. In an interior equilibrium for both communities, an individual in community $J$ must be indifferent between protecting or not. The payoff from protection is constant, and so is the payoff to an unprotected individual. The payoffs to an unprotected individual are strictly falling in the incidence, so an individual in community $J$ must be facing a constant incidence $q\left(\theta_{J}\right)$ (recall here that $q\left(\theta_{J}\right)$ denotes the equilibrium incidence in a homogenous population with type $\left.\theta_{J}\right)$. An interior equilibrium thus entails $q\left(\theta_{J}\right)=\beta Q_{-J}+(1-\beta) Q_{J}$, $J \in\{H, L\}$. Solving for $Q_{J}, J \in\{H, L\}$, yields

$$
\begin{aligned}
& Q_{H}=\frac{1}{2}\left[q\left(\theta_{L}\right)+q\left(\theta_{H}\right)+\frac{q\left(\theta_{L}\right)-q\left(\theta_{H}\right)}{2 \beta-1}\right] \\
& Q_{L}=\frac{1}{2}\left[q\left(\theta_{L}\right)+q\left(\theta_{H}\right)-\frac{q\left(\theta_{L}\right)-q\left(\theta_{H}\right)}{2 \beta-1}\right] .
\end{aligned}
$$

$Q_{H}$ and $Q_{L}$ are thus uniquely determined. Since individuals in community $J$ face $q\left(\theta_{J}\right)$, interaction of unprotected individuals in community $J$ is uniquely determined too, as is the probability of infection for these individuals. Equation (11) now determines $V_{J}$. This shows that an equilibrium that is interior for both communities is uniquely determined. 
We next establish parts 1-3 of the Proposition. We know from Proposition 3 that equilibrium incidence is falling in population type; so $q\left(\theta_{L}\right)-q\left(\theta_{H}\right)>0$. Using (13)-(14), $Q_{H}$ is therefore in equilibrium strictly decreasing in $\beta$ while $Q_{L}$ is strictly increasing. Since individuals in community $J$ face $q\left(\theta_{J}\right)$ throughout, interaction of unprotected individuals in community $J$ remains fixed (in equilibrium) as we vary $\beta$. Similarly, as we vary $\beta$, the probability that an unprotected individual becomes infected remains constant in each community. An inspection of the expression for $Q_{J}$ in (11) reveals that changes in incidence must occur entirely through adjustments in the rate of protection, $V_{J} . V_{H}$ therefore strictly increases in $\beta$ while $V_{L}$ strictly decreases. This establishes part 1 of the Proposition.

The arguments for Parts 2 and 3 of the Proposition are similar to those used in the proof of Proposition 3.

Proof of Proposition 6: $q\left(\theta_{J}\right)$, recall, denotes the equilibrium incidence of the disease in a homogenous population with type $\theta_{J}$. We first show that $q\left(\theta_{J}\right) \in(0, \epsilon)$ implies $V\left(\theta_{J}\right) \in(0,1)$, i.e. that the unique equilibrium in a homogenous population with type $\theta_{J}$ is interior. $V\left(\theta_{J}\right)=$ 1 is impossible, as this would imply $q\left(\theta_{J}\right)=0 . V\left(\theta_{J}\right)=0$, on the other hand, would imply $q\left(\theta_{J}\right)=p\left(\theta_{J}\right)$, the equilibrium probability of infection for unprotected individuals. Thus, by (3), $q\left(\theta_{J}\right) \geq \epsilon$ (recall, $\epsilon$ is the probability of contracting the disease exogenously), contradicting our initial assumption. This finishes to establish that $V\left(\theta_{J}\right) \in(0,1), J \in\{H, L\}$.

Since the unique equilibrium in a homogenous population is interior for both types, individuals of type $\theta_{J}$ are exactly indifferent between vaccinating or not when facing the incidence $q\left(\theta_{J}\right)$. By the same token, they strictly prefer vaccinating if facing $q>q\left(\theta_{J}\right)$, and strictly prefer remaining unprotected if facing $q<q\left(\theta_{J}\right)$.

As in Proposition 5, let $q_{J}$ denote the incidence facing individuals in community $J$ and $Q_{J}$ the incidence within community $J$. Thus here $q_{J}=Q_{-J}$, since an individual in community 
$J$ interacts exclusively with individuals in communities $-J$. Suppose an equilibrium exists in which some individual in community $J$ protects. We will first show that if all individuals in community $-J$ remain unprotected, then it must be that all individuals in community $J$ in fact protect. We will then show that if some individual in community $-J$ protects, then the equilibrium must be interior.

If all individuals in community $-J$ remain unprotected, each of them must be infected with probability $\epsilon$ at least, inducing $q_{J}=Q_{-J} \geq \epsilon$. But $q\left(\theta_{J}\right)<\epsilon$, so if all individuals in community $-J$ remain unprotected then all individuals in community $J$ must protect. This in turn makes it optimal for individuals in community $-J$ not to protect.

Next, suppose that some individual in $-J$ protects. We must then have $q_{-J}=Q_{J} \geq$ $q\left(\theta_{-J}\right)$. Since $q\left(\theta_{-J}\right)>0$, some individual in community $J$ must therefore be unprotected. Thus, in community $J$, some individuals protects while others do not. By symmetry, the same must then be true for community $-J$. This shows that if some individual in community $-J$ protects, then the equilibrium must be interior in both communities. Furthermore, this equilibrium is fully characterized by the condition $Q_{J}=q\left(\theta_{-J}\right), J \in\{H, L\}$. The arguments above establish that there exist exactly three equilibria, satisfying the description given in the statement of the Proposition.

Proof of welfare ranking: As in Proposition 3, let $p\left(k_{i}, q_{i}\right)$ denote the infection probability of $i$ interacting according to $k_{i}$ and facing incidence $q_{i}$. We then have

$$
\begin{gathered}
W_{1}=\frac{1}{2}\left(2 \theta_{H} g(\bar{k})-\gamma\right)+\frac{1}{2}\left[\theta_{L} g\left(k_{u}\left(\theta_{L}\right)\right)+\left(1-p\left(k_{u}\left(\theta_{L}\right), 0\right)\right) \theta_{L} g(\bar{k})\right] \\
W_{2}=\frac{1}{2}\left(2 \theta_{L} g(\bar{k})-\gamma\right)+\frac{1}{2}\left[\theta_{H} g\left(k_{u}\left(\theta_{H}\right)\right)+\left(1-p\left(k_{u}\left(\theta_{H}\right), 0\right)\right) \theta_{H} g(\bar{k})\right],
\end{gathered}
$$

where here $k_{u}\left(\theta_{J}\right)$ is used to denote the optimal interaction of unprotected individuals in 
community $J$ facing zero incidence. Thus $W_{1}-W_{2}=1 / 2\left[T\left(\theta_{H}\right)-T\left(\theta_{L}\right)\right]$, where

$$
T(\theta)=(2 \theta g(\bar{k})-\gamma)-\theta g\left(k_{u}(\theta)\right)-\left(1-p\left(k_{u}(\theta), 0\right)\right) \theta g(\bar{k})
$$

Differentiating yields

$$
\frac{d T}{d \theta}=2 g(\bar{k})-g\left(k_{u}(\theta)\right)-\left(1-p\left(k_{u}(\theta), 0\right)\right) g(\bar{k})
$$

where we have used the fact that $k_{u}(\theta)$ is optimal for an unprotected individual in community $J$ facing zero incidence. But then, clearly, $\frac{d T}{d \theta}>0$ and so $W_{1}>W_{2}$.

That both $W_{1}$ and $W_{2}$ are strictly greater than $W_{3}$ follows from the observation that $W_{3}=1 / 2\left(2 \theta_{H} g(\bar{k})-\gamma\right)+1 / 2\left(2 \theta_{L} g(\bar{k})-\gamma\right)$, while the maximum payoff attained by unprotected individuals is strictly greater when facing zero incidence than when facing $q\left(\theta_{J}\right)>0$.

\section{References}

1. Alpcan, T. and T. Basar(2011), Network Security: A Decision and Game Theoretic Approach. Cambridge University Press. Cambridge, England.

2. Anderson, R. (2008), Security Engineering. Second Edition. Wiley.

3. Anderson, R., Bohme, R., Clayton, R., and T. Moore (2007), Security economics and the internal market, European Network and Information Security Agency.

4. Anderson R. M., and R.M. May (1991), Infectious Diseases of Humans: Dynamics and Control. OUP, Oxford. 
5. Aspnes, J., K. Chang, A. Yampolskiy (2006), Inoculation strategies for victims of viruses and the sum-of-squares partition problem, Journal of Computer and System Sciences,

6. Brito, D.L., E. Sheshinski and M.D. Intriligator (1991), Externalities and compulsory vaccinations, Journal of Public Economics, 45, 69-90.

7. Chen, F., and F. Toxvaerd (2014), The economics of vaccination, Mimeo, Cambridge University.

8. Center for Strategic and International Studies (2013), The Economic Impact of Cybercrime and Cyber Espionage.

9. Detica and Cabinet Office (2011), The Cost of Cyber Crime.

10. Francis, P. J. (1997), Dynamic epidemiology and the market for vaccination, Journal of Public Economics, 63, 3, 383-406.

11. Galeotti, A., and B. Rogers (2013), Strategic Immunization and Group Structure, American Economic Journal-Micro, 5, 2, 1-32.

12. Geoffard, P-Y., and T. Philipson (1996), Rational Epidemics and Their Public Control, International Economic Review, 37, 3, 603-624.

13. Geoffard, P-Y., and T. Philipson (1997), Disease eradication: private versus public vaccination, American Economic Review, 87, 1, 222-230.

14. Gersovitz, M. (2011), The economics of infection control, Annual Review Resource Economics, 3, 27796

15. Gersovitz, M., and J. S. Hammer (2004), The economical control of infectious diseases, Economic Journal, 114, 1-27. 
16. Goldman, S. M. and J. Lightwood (2002), Cost optimization in the SIS model of infectious disease with treatment, Topics in Economic Analysis and Policy, 2, 1, 1-22.

17. Hays, J. N. (2009), The Burdens of Disease: Epidemics and Human Response in Western History. Rutgers University Press.

18. Kremer, M. (1996), Integrating Behavioral Choice into Epidemiological Models of AIDS, Quarterly Journal of Economics, 111, 2, 549-73.

19. Moore, T., Clayton, R., and R. Anderson (2011), Economics and Internet Security: a Survey of Recent Analytical, Empirical and Behavioral Research Harvard University Computer Science Department, Discussion Paper TR-03-11.

20. Philipson, T. and R. Posner (1993), Private Choices and Public Health: The AIDS Epidemic in an Economic Perspective. Harvard University Press.

21. World Health Organization (2013), HIV/AIDS. Fact Sheet Number 2013.

22. World Health Organization (2014a), Influenza (Seasonal). Fact Sheet Number 211.

23. World Health Organization (2014b), Tuberculosis. Fact Sheet Number 104. 